\title{
Cutaneous mucormycosis: an unusual cause of decompensation in a patient with ethanol-related cirrhosis with COVID-19 exposure
}

\author{
Sherna Menezes, ${ }^{1}$ Janu Santhosh Kumar, ${ }^{2}$ Omkar S Rudra, ${ }^{3}$ Aabha Nagral (i) ${ }^{1}$
}

'Department of

Gastroenterology, Jaslok

Hospital and Research Centre,

Mumbai, Maharashtra, India

${ }^{2}$ Department of Medicine, Jaslok

Hospital and Research Centre,

Mumbai, Maharashtra, India

${ }^{3}$ Department of Critical Care

Jaslok Hospital and Research

Centre, Mumbai, Maharashtra,

India

Correspondence to

Dr Aabha Nagral;

aabhanagral@gmail.com

Accepted 20 January 2022

Check for updates

(c) BMJ Publishing Group

Limited 2022. No commercial

re-use. See rights and

permissions. Published by BMJ.

To cite: Menezes $\mathrm{S}$,

Kumar JS, Rudra OS,

et al. BMJ Case Rep

2022:15:e247399.

doi:10.1136/bcr-2021-

247399

\section{SUMMARY}

We describe a case of cutaneous mucormycosis in a middle-aged man with ethanol-related chronic liver disease. He presented with symptoms of fever, breathlessness for 10 days and altered mental status for 2 days. On admission, he was in septic shock and had acute respiratory distress syndrome (ARDS). He was noted to have ruptured blisters in his left axilla. Although he repeatedly had negative COVID-19 Reverse Transcription-PCR results, he had positive IgG antibodies for COVID-19. He was managed with broad-spectrum antibiotics, steroids, vasopressors and ventilation for ARDS. Over the course of his hospitalisation, the axillary lesion progressed to a necrotising ulcer with deep tissue invasion. Debridement and culture of the axillary ulcer revealed mucor species, and he was started on amphotericin and posaconazole for mucormycosis. Unfortunately, he continued to deteriorate despite aggressive management and died after a prolonged hospital stay of 40 days.

\section{BACKGROUND}

Mucormycosis is a rare opportunistic fungal infection that causes angio-invasive symptoms leading to tissue necrosis and eschar formation, most commonly in the rhino-orbital-cerebral region. It has been reported with increasing frequency in recent months due to the suppression of the immune system in COVID-19 infection as well as the increased usage of corticosteroids in its management. The association of COVID-19 with fungal coinfections, including mucormycosis, invasive aspergillosis and candidiasis, has been well established. ${ }^{1-8}$ Patients with decompensated liver cirrhosis are at an increased risk of invasive fungal infections due to various factors seen in cirrhosis, including immune deficiency, malnutrition and frequent hospitalisations with invasive procedures. Patients with cirrhosis with exposure to COVID-19, in particular, would be expected to be at further risk of developing fungal infections due to the added immunosuppression induced by this infection. We describe an unusual site of invasive mucormycosis presenting as a cause of acute decompensation of chronic liver disease.

\section{CASE PRESENTATION}

A middle-aged man, a known case of compensated ethanol-related chronic liver disease, was referred to our hospital for persistent high-grade fever, breathlessness for 10 days and altered sensorium for 2 days. He has had a history of hypertension for 12 years and was diagnosed with cirrhosis 3 years ago. On presentation, he was drowsy, but irritable and agitated on arousal. He was hypotensive with a blood pressure of $80 / 50 \mathrm{~mm} \mathrm{Hg}$, a pulse of 78 beats/min, a respiratory rate of $28 / \mathrm{min}$ and an $\mathrm{SpO}_{2}$ of $96 \%$ on $4 \mathrm{~L}$ of oxygen. He was febrile, pale, deeply icteric with dependent oedema and ruptured blisters with ulcers in the left axilla. The remainder of the examination was unremarkable.

\section{INVESTIGATIONS}

On initial blood investigations, his haemoglobin was $71 \mathrm{~g} / \mathrm{L}$, his white cell count was $5.77 \times 10^{9} / \mathrm{L}$ with $88 \%$ neutrophils and $8 \%$ lymphocytes, and his platelet count was $17 \times 10^{9} / \mathrm{L}$. His fasting blood glucose at admission was elevated to $191 \mathrm{mg} / \mathrm{dL}$. His liver function tests showed total bilirubin of $62.6 \mathrm{~mol} / \mathrm{L}$ with a direct bilirubin of $45.8 \mathrm{~mol} / \mathrm{L}$, Serum glutamic oxaloacetic transaminase (SGOT) 573.7 U/L, Serum glutamic pyruvic transaminase (SGPT) $95 \mathrm{U} / \mathrm{L}$, albumin $15 \mathrm{~g} / \mathrm{L}$, ammonia $56.35 \mathrm{~mol} / \mathrm{L}$, and alkaline phosphatase $132 \mathrm{U} / \mathrm{L}$. His coagulation studies were abnormal with a prothrombin time (PT) of $37.4 \mathrm{~s}$ and an International Normalized Ratio (INR) of 3.21. His ChildTurcotte-Pugh criteria score (CTP score) was 14 based on the physical examination and initial lab tests. A nasopharyngeal swab for SARS-CoV-2 Reverse Transcription-PCR was negative and he underwent an high-resolution computed tomography (HRCT) of the chest which was indeterminate for a COVID-19 infection (CORADS 3). Due to difficult weaning, worsening lung shadows and copious tracheal secretions, repeated COVID-19 RT-PCR tests were sent during the initial week, which came back negative. His COVID-19 IgG antibody test, however, came positive on day 7 of hospitalisation. The blood and urine aerobic and fungal cultures during the first week of admission showed no growth. His ventilatory requirements increased and he needed to be intubated. In the second week of hospitalisation, a bronchoscopy with the culture of bronchoalveolar lavage was done, which showed scanty growth of Candida albicans with sensitivity to most antifungals (amphotericin, caspofungin, fluconazole, micafungin, voriconazole). Three weeks after admission, extension and necrosis of the left axillary ulcer was noticed. Imaging was performed and revealed its extension into the muscular plane, abutting the axillary vein. The intraoperative sample obtained after debridement was sent 
for histopathology. The report showed a dense mixed inflammatory infiltrate and many non-septate thick foldable periodic acid-Schiff (PAS) and Grocott methenamine silver (GMS) stainpositive fungal filaments with wide-angle branching suggestive of mucormycosis. The pus obtained from the axillary wound was cultured and grew extended-spectrum beta-lactamase Escherichia coli and vancomycin-resistant Enterococcus.

\section{TREATMENT}

On admission, he was started on a norepinephrine infusion and oxygen support. He was empirically started on broad-spectrum antibiotics (piperacillin-tazobactam), pending blood culture reports. Anti-encephalopathy medications, multiple blood transfusions and other supportive measures were given during admission. As his condition deteriorated, he was mechanically ventilated. Antibiotics were further escalated. Diuretic infusions and terlipressin were initiated as he was oliguric and hepatorenal syndrome was suspected. His liver function tests showed a mild improvement with the treatment on day 15. A total bilirubin of $46.5 \mathrm{~mol} / \mathrm{L}$ with a direct bilirubin of $34.8 \mathrm{~mol} / \mathrm{L}$, SGOT of $395 \mathrm{U} / \mathrm{L}$, SGPT of $70 \mathrm{U} / \mathrm{L}$, albumin of $3.2 \mathrm{~g} / \mathrm{L}$, ammonia of $43 \mathrm{~mol} / \mathrm{L}$, alkaline phosphatase of $126 \mathrm{U} / \mathrm{L}, \mathrm{PT}$ of $27 \mathrm{~s}$ and an INR of 2.8 were recorded (CTP score was 10 points). Fluconazole was initiated after Candida growth was observed on tracheal aspirates but later escalated to anidulafungin. Over the course of admission, his fractional inspired oxygen, as well as positive endexpiratory pressure (PEEP) requirement, steadily increased, and his inotrope requirement also increased, hence a decision was taken to start him on intravenous steroids. Repeat CT imaging of the chest was suggestive of acute respiratory distress syndrome (ARDS). His repeated liver function tests began to show a decline over the third week of treatment. A surgical referral was sent for the necrotic axillary lesion, and debridement with vacuum-assisted closure of the wound was performed on day 22 of hospitalisation. Intraoperatively, it was observed that there was encroachment of the axillary vein wall, which was removed, and the defect sutured (figure 1). Liposomal amphotericin B with posaconazole was initiated for the mucormycosis. Antibiotics were modified as per the wound culture sensitivity reports.

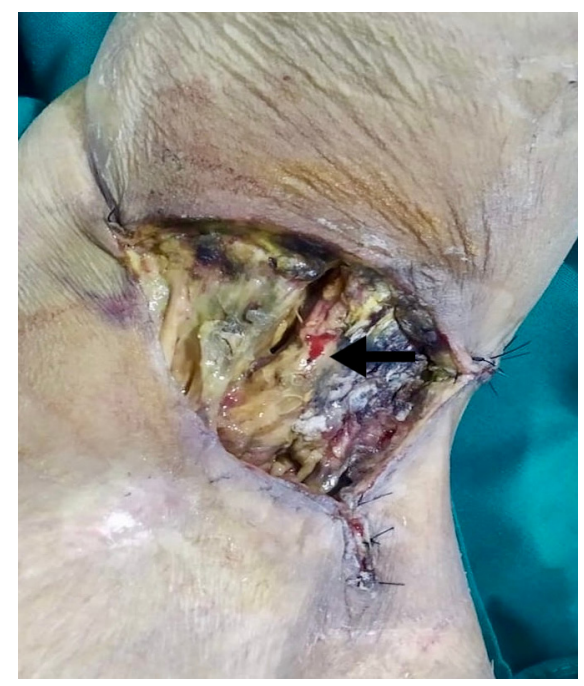

Figure 1 Intraoperative image of the left axilla demonstrating the creeping necrosis that is classic of invasive mucormycosis and the involvement of the axillary vein (black arrow).

\section{OUTCOME AND FOLLOW-UP}

Almost a month after admission, he continued to have worsening sepsis with shock, needing higher inotropic doses. The axillary wound showed more slough and was re-explored. However, he continued to deteriorate and had increasing requirements for vasopressors and higher antibiotics. His LFTs also showed worsening with a total bilirubin of $70.4 \mathrm{~mol} / \mathrm{L}$ and a direct bilirubin of $59.8 \mathrm{~mol} / \mathrm{L}$, SGOT $689 \mathrm{U} / \mathrm{L}$, SGPT $178 \mathrm{U} / \mathrm{L}$, albumin $18 \mathrm{~g} / \mathrm{L}$ and alkaline phosphatase $176 \mathrm{U} / \mathrm{L}$. His coagulation studies showed a PT of $40 \mathrm{~s}$ and an INR of 3.7 (CTP score of 15 points). His worsening acute kidney injury with hypotension necessitated the initiation of continuous renal replacement therapy. A few days later, unfortunately, he succumbed to a bradycardic cardiac arrest.

\section{DISCUSSION}

Mucormycosis is the third most common invasive fungal infection, seen after aspergillosis and candidiasis. These fungi belong to the order Mucorales and are ubiquitous in nature. They are usually seen in immunocompromised people and gain entry into the body by inhalation, ingestion or inoculation of spores. The predisposing conditions for the development of mucormycosis include poorly controlled diabetes with or without diabetic ketoacidosis (especially for the rhino-orbito-cerebral presentation), malignant haematological disease, prolonged and severe neutropenia, iron overload, major trauma, corticosteroid usage and illicit intravenous drug use. ${ }^{9}$ Our patient had exposure to COVID-19 with suggestive symptoms and a positive antibody test, though his COVID-19 RT-PCR tests were negative. He also had ethanol-related liver cirrhosis and was diagnosed as diabetic on admission. We believe all these factors played a role in the causation of mucormycosis.

The risk factors for cutaneous presentation include disrupted skin barriers due to burns, trauma, intravascular devices or catheters, and also due to maceration of the skin leading to inoculation of the fungal spores in the skin. ${ }^{10}$ In an observational study published by Patel et al, among 465 mucormycosis cases without COVID-19 in India, rhino-orbital mucormycosis was the most frequently observed presentation $(67.7 \%)$, followed by pulmonary $(13.3 \%)$ and cutaneous $(10.5 \%) .{ }^{11}$

The incidence of mucormycosis has rapidly increased during the COVID-19 pandemic. A systematic review of 101 mucormycosis cases seen in COVID-19 reported that diabetes mellitus was present in $80 \%$ of cases, and corticosteroids were given in $76.3 \%$ of cases. ${ }^{7}$ The review reported only a single case of cutaneous mucormycosis out of the 101 cases.

Cutaneous mucormycosis in COVID-19 is extremely rare. A case of upper extremity cutaneous mucormycosis infection at the site of intravenous access was seen in a heart transplant patient after recovering from COVID-19. ${ }^{12}$ Another case of cutaneous and rhino-orbito-cerebral mucormycosis was reported in a 59-year-old uncontrolled diabetic 3 weeks after recovering from COVID-19. ${ }^{13}$ None of these patients survived, despite aggressive management.

There are very few cases of cutaneous mucormycosis reported in patients with liver cirrhosis. ${ }^{14-17}$ In three of the cases, the aetiology of cirrhosis was alcoholic liver disease. ${ }^{1415} 17$ All four cases reported the outcome as death. In most of these cases, an initial traumatic insult to the area was present. The typical sign of the cutaneous presentation is a necrotic eschar with surrounding erythema and induration. In our case, the patient presented with hepatic encephalopathy with respiratory failure, and an axillary ulcer without any necrotic tissue was incidentally noticed 
without any obvious history of trauma to the area. There was a delay in detecting mucormycosis due to the unlikely location of the lesion and concomitant severe septic presentation and ARDS. The fungal blood cultures were negative in the first week since the initial infection was most probably limited to the cutaneous area. However, the infection flared with extensive deep tissue invasion, likely due to the administration of steroids for septic shock. Urgent surgical debridement was performed and rapid treatment with antifungals was initiated after histopathological diagnosis of mucormycosis. The Candida in the tracheal culture seemed to be a secondary event to a possible aspiration due to his altered sensorium.

In conclusion, cutaneous mucormycosis needs to be aggressively managed at an early stage as it can be a rapidly fatal fungal infection with $100 \%$ mortality in patients with cirrhosis, especially in patients like ours with additional predisposing factors like COVID-19 sepsis and parenteral steroid administration. Early detection of the infection is the key to treatment, and identification of infection in high-risk patients is essential, especially those with recent exposure to COVID-19. Control of risk factors, corticosteroid regulation and a low threshold for diagnosis will aid in the prevention of mucormycosis. In the setting of super-added causes of immunosuppression, fungal infections can become a cause of acute decompensation in patients with chronic liver disease.

\section{Learning points}

- Chronic liver disease may decompensate due to any infection, including invasive fungal infections.

- Multiple risk factors for reduced immunity such as cirrhosis, COVID-19 exposure, diabetes and steroid exposure serve as a rich milieu for invasive fungal infections.

- A high index of clinical suspicion for cutaneous mucormycosis should be present in a high-risk case with a non-healing skin wound.

Contributors SM contributed to the acquisition and interpretation of data and writing of the manuscript. JSK contributed to the acquisition of data and editing of the manuscript. OSR contributed to the design of the article and editing of the manuscript. AN contributed to the conception and design of the article, editing and critical revision of the manuscript. All authors have read, edited and approved the final document prior to submission.

Funding The authors have not declared a specific grant for this research from any funding agency in the public, commercial or not-for-profit sectors.

Competing interests None declared.
Patient consent for publication Next of kin consent obtained.

Provenance and peer review Not commissioned; externally peer reviewed.

Case reports provide a valuable learning resource for the scientific community and can indicate areas of interest for future research. They should not be used in isolation to guide treatment choices or public health policy.

\section{ORCID iD}

Aabha Nagral http://orcid.org/0000-0003-0005-2508

\section{REFERENCES}

1 White PL, Dhillon R, Cordey A, et al. A national strategy to diagnose coronavirus disease 2019-Associated invasive fungal disease in the intensive care unit. Clin Infect Dis 2021;73:ciaa1298:e1634-44.

2 Bartoletti M, Pascale R, Cricca M, et al. Epidemiology of invasive pulmonary aspergillosis among intubated patients with COVID-19: a prospective study. Clin Infect Dis 2021;73:ciaa1065:e3606-14.

3 Machado M, Valerio M, Álvarez-Uría A, et al. Invasive pulmonary aspergillosis in the COVID-19 era: an expected new entity. Mycoses 2021;64:132-43.

4 Chong WH, Neu KP, Incidence NKP. Incidence, diagnosis and outcomes of COVID19-associated pulmonary aspergillosis (CAPA): a systematic review. J Hosp Infect 2021:113:115-29.

5 Lai C-C, Yu W-L. COVID-19 associated with pulmonary aspergillosis: a literature review. J Microbiol Immunol Infect 2021;54:46-53.

6 Arastehfar A, Carvalho A, Nguyen MH, et al. COVID-19-Associated candidiasis (CAC): an underestimated complication in the absence of immunological predispositions? $J$ Fungi 2020;6:211.

7 Singh AK, Singh R, Joshi SR, et al. Mucormycosis in COVID-19: a systematic review of cases reported worldwide and in India. Diabetes Metab Syndr 2021;15:102146.

8 Kayaaslan B, Eser F, Kaya Kalem A, et al. Characteristics of candidemia in COVID-19 patients; increased incidence, earlier occurrence and higher mortality rates compared to non-COVID-19 patients. Mycoses 2021;64:1083-91.

9 Petrikkos G, Skiada A, Lortholary 0, et al. Epidemiology and clinical manifestations of mucormycosis. Clin Infect Dis 2012;54 Suppl 1:S23-34.

10 Spellberg B, Edwards J, Ibrahim A. Novel perspectives on mucormycosis: pathophysiology, presentation, and management. Clin Microbiol Rev 2005; 18:556-69.

11 Patel A, Kaur H, Xess I, et al. A multicentre observational study on the epidemiology, risk factors, management and outcomes of mucormycosis in India. Clin Microbiol Infect 2020:26:944.e9-944.e15.

12 Khatri A, Chang K-M, Berlinrut I, et al. Mucormycosis after Coronavirus disease 2019 infection in a heart transplant recipient - Case report and review of literature. J Mycol Med 2021;31:101125.

13 Roushdy T, Hamid E. A case series of post COVID-19 mucormycosis-a neurological prospective. Egypt J Neurol Psychiatr Neurosurg 2021;57:100.

14 Raizman NM, Parisien M, Grafe MW, et al. Mucormycosis of the upper extremity in a patient with alcoholic encephalopathy. J Hand Surg Am 2007;32:384-8.

15 Wollstein R, Palekar A. Mucormycosis infection following intravenous access in the forearm. Can J Plast Surg 2010;18:e30:30-2.

16 Elsiesy $H$, Saad M, Shorman M, et al. Invasive mucormycosis in a patient with liver cirrhosis: case report and review of the literature. Hepat Mon 2013;13:e10858.

17 Kanagaraju V, Narayanasamy VK, Sukumaran S, et al. Invasive, gangrenous mucormycosis of arm: a fatal opportunistic infection in a highly immunocompromised host. Indian J Pathol Microbiol 2019;62:618-20.

Copyright 2022 BMJ Publishing Group. All rights reserved. For permission to reuse any of this content visit

https://www.bmj.com/company/products-services/rights-and-licensing/permissions/

BMJ Case Report Fellows may re-use this article for personal use and teaching without any further permission.

Become a Fellow of BMJ Case Reports today and you can:

- Submit as many cases as you like

- Enjoy fast sympathetic peer review and rapid publication of accepted articles

- Access all the published articles

- Re-use any of the published material for personal use and teaching without further permission

Customer Service

If you have any further queries about your subscription, please contact our customer services team on +44 (0) 2071111105 or via email at support@bmj.com.

Visit casereports.bmj.com for more articles like this and to become a Fellow 